- Nota -

\title{
EL MECANISMO PARA LA REDUCCIÓN DE LA DEFORESTACIÓN Y LA DEGRADACIÓN FORESTAL Y PARA EL FOMENTO DE LAS RESERVAS DE CARBONO (REDD+): NOTA SOBRE UN INNOVADOR INSTRUMENTO DE MITIGACIÓN
}

\author{
ALEJANDRA TORRES CAMPRUBí \\ Becaria de investigación del Área de Derecho Internacional Público \\ Universidad Autónoma de Madrid \\ alejandra.torres@uam.es
}

Recibido: 27 de octubre de 2010 / Aceptado: 15 de noviembre de 2010

RESUMEN: A través de un nuevo mecanismo de naturaleza financiera, conocido en la actualidad por sus siglas como REDD+, el sector forestal se ha convertido en uno de los ámbitos con mayor grado de desarrollo en las negociaciones, todavía en curso, en torno al futuro del régimen del cambio climático para el período posterior al 2012. Este innovador instrumento de mitigación tendrá por objetivo incentivar la reducción de emisiones de carbono provenientes de la deforestación y la degradación forestal, así como fomentar el desarrollo de sumideros de carbono. Tras recordar la relevancia científica de la deforestación en el régimen del cambio climático (I), esta nota explicará el alcance, los objetivos y la ubicación del REDD+ en la arquitectura del régimen (II). Asimismo, trazará brevemente la evolución del REDD+ a lo largo de las principales etapas de su negociación (III), sintetizando para concluir los puntos más controvertidos que permanecen pendientes de esclarecimiento con vistas a la próxima Cumbre del Clima de Cancún en el 2010 (IV).

RESUM: Mitjançant el nou mecanisme de naturalesa financera, conegut en l'actualitat per les sigles REDD+, el sector forestal ha esdevingut un dels àmbits amb més grau de desenvolupament en les negociacions, encara en curs, del futur règim del canvi climàtic per al període posterior al 2012. Aquest innovador instrument de mitigació tindrà per 
objectiu incentivar la reducció d'emissions de carboni provinents de la desforestació i la degradació forestal, així com fomentar el desenvolupament d'embornal de carboni. Després de recordar la rellevància científica de la desforestació en el règim del canvi climàtic (I), aquesta nota explicarà l'abast, els objectius i la ubicació del REDD+ en l'arquitectura del règim (II). Així mateix, traçarà breument l'evolució del REDD+ a través de les diferents etapes de la negociació (III), tot sintetitzant, per concloure, els punts més controvertits que resten pendents d'aclarir a la cimera del clima de Cancún el 2010 (IV).

ABSTRACT: As a result of the new financial mechanism known as 'REDD+', the forestry sector is now playing a central role in the on-going negotiations on the future of the post-2012 climate change regime. The aim of this innovative mitigation mechanism is to encourage the reduction of carbon emissions from deforestation and forest degradation, and to promote the development of carbon sinks. After summarizing the scientific importance of integrating deforestation into the climate change regime (I), this Note will explain the scope and objectives of REDD+ and its position within the climate change architecture (II). It will also briefly describe the evolution of REDD + through the various stages of negotiation (III), and it will conclude by synthesizing the most controversial issues that still need to be discussed at the next 2010 Climate Change Summit in Cancun (IV).

PALABRAS CLAVE: mecanismo financiero REDD + - deforestación - cambio climático - mitigación

PARAULES CLAU: mecanisme financer REDD + - desforestació - canvi climàtic — mitigació

KEY WORDS: REDD+ financial mechanism - deforestation - climate change mitigation 
Sumario: I. Introducción. Deforestación y cambio climático: dos fenómenos asociados. II. Elementos básicos de la configuración del mecanismo REDD+. 1. Alcance y objetivos del REDD+. 2. Localización del REDD+ en la estructura normativa del régimen del cambio climático. 3. Distinción entre el REDD+ y las actividades LULUCF incorporadas previamente a los mecanismos flexibles del Protocolo de Kioto. III. La historia de REDD+: principales etapas de su negociación internacional. 1. Bali 2007: orígenes de la propuesta de creación del mecanismo. 2. Poznan 2008: el impulso del desarrollo de proyectos piloto. 3. Copenhague 2009: avances en la operatividad del mecanismo. IV. Conclusiones: incógnitas pendientes sobre el funcionamiento del REDD+ con vistas a la Cumbre del Clima de Cancún 2010 (COP-16)

\section{INTRODUCCIÓN. DEFORESTACIÓN Y CAMBIO CLIMÁTICO: DOS FENÓMENOS ASOCIADOS}

Desde que en el 2007 el Panel Intergubernamental sobre Cambio Climático (IPCC) publicara su Cuarto Informe de Evaluación $(\mathrm{AR} 4)^{1}$, la importancia y relevancia del papel del sector forestal en el régimen del cambio climático ha dado sin duda un giro importante, al pasar a formar parte de la agenda de este con una firmeza hasta entonces desconocida. Dicho informe estableció entonces una serie de datos que permitieron visualizar tanto la magnitud de la presencia científica de los bosques en el ciclo del carbono como el interés económico, ecológico y social que su tratamiento en el régimen del cambio climático podía llegar a suponer² .

Las cifras dibujaban la situación del siguiente modo. En primer lugar, el 30\% de la superficie terrestre global lo constituyen áreas forestales, que representan alrededor de 4.000 millones de hectáreas principalmente localizadas en las zonas tropicales de América de Sur, África y el sudeste asiático. No obstante, a pesar de la amplia extensión de dichas áreas, estas se ven constantemente amenazadas por prácticas de deforestación que buscan transformarlas en terrenos cultivables, extender la presencia de asentamientos e infraestructuras, y responder a la demanda mundial del mercado sobre productos forestales como la madera o la fibra. Así, la deforestación supone en la

\footnotetext{
${ }^{1}$ IPCC, 2007, Cuarto Informe de Evaluación (Fourth Assessment Report), recuperado el 25 de octubre de 2010, disponible en inglés en: http://www.ipcc.ch/pdf/assessment-report/ar4/syr/ar4 syr.pdf

${ }^{2}$ Los detalles que se citan a continuación proceden concretamente del informe IPCC, 2007, Forestry in Climate Change 2007: Mitigation. Contribution of Working Group III to the Fourth Assessment Report of the Intergovernmetnal Panel on Climate Change, recuperado el 25 de octubre de 2010, disponible en: http://www.ipcc.ch/ipccreports/ar4-wg3.htm
} 
actualidad una pérdida anual de alrededor 12,9 millones de hectáreas de bosques ${ }^{3}$, pérdida que pone en riesgo la sostenibilidad de los ecosistemas forestales concernidos.

Más interesante aún es el impacto de dichos índices de deforestación en el fenómeno del cambio climático, y del que se deriva la relevancia de la integración del sector forestal en el régimen normativo internacional sobre este fenómeno. La deforestación provoca la emisión tanto de dióxido de carbono (producida por la tala y quema de bosques), como de nitrógeno óxido (generada por la utilización de abonos y la quema de masa biótica). El informe señala así que en el sector forestal se origina el 17,4\% del total anual de las emisiones de gases de efecto invernadero ${ }^{4}$.

La inclusión de la deforestación en la agenda del cambio climático se remonta a la Conferencia de las Partes del Convenio de Naciones Unidas para el Cambio Climático (en adelante COP), realizada en Bali (Indonesia) en el 2007 (COP-13). Netamente influenciadas por el informe del IPCC publicado el mismo año, las partes del Convenio Marco de Naciones Unidas sobre el Cambio Climático (CMNUCC ${ }^{5}$ ) adoptaron, a propuesta de Costa Rica y Papúa Nueva Guinea (que actuaban en nombre de la Coalition of Rainforest Nations ${ }^{6}$ ), la Decisión 2/COP-137 ${ }^{7}$ Dicha decisión establecía el objetivo de crear un nuevo mecanismo financiero destinado a incentivar la reducción en países en desarrollo de las emisiones provenientes de la deforestación.

Tras haber recordado en este primer epígrafe el impacto científico de la deforestación en las emisiones anuales de carbono, esta nota explica los elementos básicos de la

${ }^{3}$ Ibíd. p. 544. Esta cifra fue calculada para el período 2000-2005, que indica un ligero descenso con relación a las cifras de los años 1990. Por su parte, el programa de Naciones Unidas sobre el mecanismo REDD + cifra en 13 millones de hectáreas la pérdida anual de superficie forestal por deforestación entre 1990 y 2005. UN-REDD Program, 2008, Framework Document, recuperado el 25 de octubre de 2010, disponible en: http://www.un-redd.org/Publications/tabid/587/Default.aspx

${ }^{4}$ Véase nota n. ${ }^{\circ} 1$ supra, p. 5.

5 Convenio Marco de Naciones Unidas sobre el Cambio Climático, adoptado en la Conferencia de Naciones Unidas sobre Medio Ambiente y Desarrollo (Río de Janeiro), entre el 4 y el 14 de junio de 1992.

${ }^{6}$ Página web de la Coalition for Rainforest Nations, recuperado el 25 de octubre de 2010, disponible en: http://www.rainforestcoalition.org/eng/

7 Decisión 2/COP.13, "Reducción de las emisiones derivadas de la deforestación en los países en desarrollo: métodos para estimular la adopción de medidas", FCCC/CP/2007/6/Add.1, p. 8. Recuperado el 25 de octubre de 2010, texto en español disponible en:

http://unfccc.int/documentation/decisions/items/3597.php?such=j\&volltext=/CP.13\#beg 
configuración del mecanismo, así como su ubicación en el complejo entramado normativo del régimen internacional sobre cambio climático y su diferenciación respecto a otros mecanismos preexistentes que también afectan al sector forestal (II). A la luz del carácter "vivo" del mecanismo, que ha evolucionado con cada etapa de las negociaciones internacionales todavía en curso (III), la nota concluye sintetizando los puntos de inflexión relativos al futuro funcionamiento del mecanismo, que serán abordados próximamente en la Cumbre del Clima de Cancún (IV).

\section{ELEMENTOS BÁSICOS DE LA CONFIGURACIÓN DEL MECANISMO REDD+}

\section{Alcance y objetivos del REDD+}

Este innovador mecanismo de naturaleza financiera se conoce por sus siglas en inglés REDD+, que aluden al objetivo inicial del mecanismo de Reducir las Emisiones provenientes de la Deforestación y la Degradación Forestal.

Desde la adopción de la Decisión 2/COP.13 del 2007, el alcance material del mecanismo se ha ido ampliando a lo largo de las distintas etapas de negociación. Si bien en la propuesta inicial de Costa Rica y Papúa Nueva Guinea el mecanismo financiero solo tenía por objeto la reducción de emisiones provenientes de la deforestación (RED), se entendió rápidamente que las emisiones provenientes de la degradación forestal también debían ser incluidas (lo que daba paso a REDD). Finalmente, en un último paso en las negociaciones de Copenhague 2009, se extendió aún más el alcance del mecanismo, por lo que se añadió el "plus" (REDD+). Esta última extensión abarca prácticas de conservación, gestión forestal sostenible e impulso de las reservas forestales de carbono (también conocidas como sumideros de carbono).

Una de las deficiencias del texto de negociación consiste en que hoy por hoy no ofrece ninguna definición de los términos deforestación, degradación forestal, conservación forestal, gestión forestal sostenible ni fomento de las reservas de carbono. No obstante, es posible colmar esta laguna ateniéndose a las definiciones comúnmente aceptadas en la práctica sobre cada tipo de activad.

Así, se entiende por deforestación la conversión inducida por el hombre de un área forestal en terrenos de uso no forestal, generalmente ejecutada por medio de la tala de 
bosques (land-clearing). Por su parte, la degradación forestal alude a la reducción (que no conversión ni transformación) de la masa biótica de los bosques derivada de la siega o recolección no sostenibles del terreno ${ }^{8}$.

Asimismo, la reciente extensión del mecanismo para la inclusión de prácticas de conservación forestal, gestión sostenible de los bosques y fomento de sumideros de carbono (carbon sinks) implica que en la actualidad REDD+ busca no solo fomentar la reducción de las tasas de pérdida adquirida de masa forestal biótica, sino también impulsar el crecimiento de prácticas de conservación sobre bosques todavía existentes.

Por tanto, cabe indicar que se entiende por conservación la preservación de áreas forestales que, si bien en la actualidad no se encuentran en peligro de desaparición por deforestación o degradación, posiblemente lo estén en el futuro. Por su parte, la inclusión de la gestión forestal sostenible responde a la idea de que la explotación de las áreas forestales destinadas a la producción de madera se lleve a cabo de modo que la política de gestión refleje un equilibrio entre sus objetivos económicos, sociales y medioambientales.

Por último, queda por indicar el significado de la categoría más difusa, referente a las actividades dirigidas a "impulsar el desarrollo de los sumideros forestales de carbono". Esta categoría permite la inclusión de la restauración forestal, la forestación y la reforestación. Se entiende por forestación la creación de un bosque allí donde no ha existido al menos en los últimos 50 años, mediante actividades humanas como la plantación, la siembra o el fomento de semilleros naturales. El término reforestación se refiere a la creación de un bosque, mediante estos mismos tipos de actividades, en tierras originariamente boscosas que fueron posteriormente deforestadas ${ }^{9}$. Por su parte, la restauración forestal alude al proceso de ayudar a la recuperación de stocks de carbono de un bosque, todavía existente, pero que ha sido degradado o dañado.

\footnotetext{
${ }^{8}$ Véase nota.$^{\circ} 2$ supra, p. 550.

9 Véase la página del Ministerio de Medio Ambiente y Medio Rural y Marino, recuperado el 23 de octubre de 2010, disponible en:

http://www.mma.es/portal/secciones/cambio_climatico/areas_tematicas/flexibilidad/sumideros/
} 
A pesar de la complejidad y la confusión creadas por la ampliación del alcance del mecanismo, la idea de base que cimentó la configuración del mecanismo REDD + ha permanecido. Esta consistiría en crear un mecanismo que permita dar un valor financiero al "mantenimiento en pie" de árboles ya existentes (standing trees), de modo que aquellos Estados que obtengan reducciones certificadas de emisiones provenientes de la realización de actividades cubiertas por el mecanismo puedan recibir "retribución" económica a cambio.

Conviene señalar que el objetivo del mecanismo, como su nombre claramente indica, es la "reducción" de las emisiones provenientes del sector forestal, y no así su reducción excesiva ni, a fortiori, su eliminación. Tal y como recalca el Informe del Tercer Grupo de Trabajo del IPCC mencionado en esta introducción ${ }^{10}$, una excesiva reducción de los índices de deforestación provocaría una caída de la disponibilidad de productos forestales en el mercado, lo que impulsaría a su vez la demanda de productos de sustitución de origen industrial (como por ejemplo el aluminio y el plástico), generadores de más emisiones de carbono que los del sector forestal.

REDD+ ofrecería, por tanto, al menos desde una perspectiva hipotética, varios tipos de beneficios. El primero y sin duda más obvio es el beneficio ecológico derivado de la reducción de la segunda fuente de emisiones de gases de efecto invernadero más importante, por detrás de la energía y la industria $(45,3 \%$ conjuntamente) y por delante del transporte $(13,1 \%)$, lo que permitiría avanzar en la lucha global contra el cambio climático $^{11}$.

Asimismo, el REDD+ puede resultar igualmente interesante desde una perspectiva ambiental paralela, como potencial portador de beneficios añadidos en materia de

\footnotetext{
${ }^{10}$ Véase nota n. ${ }^{\circ} 2$ supra, p. 549.

11 Véase el siguiente informe, Nature Conservancy, CORTEZ R. and STEPHEN P. eds., 2009, Introductory Course on Reducing Emissions from Deforestation and Forest Degradation and the Role of Conservation, Sustainable Management of Forest and Enhancement of Forest Carbon Stocks (REDD+): A Participant Resource Manual, Updated April 2009, p.15 (cifras presentadas sobre la base de los resultados del Cuarto Informe de Evaluación del IPCC), recuperado el 23 de octubre de 2010, disponible en: www.nature.org/initiatives/climatechange/files/redd_training_manual.pdf
} 
protección de la biodiversidad ${ }^{12}$. La extensión del alcance del mecanismo para incluir el papel de la conservación, la gestión sostenible de los bosques y la promoción de stocks forestales de carbono como resultado de la Cumbre de Copenhague de 2009 no solo fortalece este beneficio añadido, sino que también refuerza el objetivo general a largo plazo del régimen de promover políticas de desarrollo "verdes" (es decir, de bajo nivel de consumo de carbono).

La evolución del REDD+ ha sido igualmente relevadora del carácter de la complejidad del mecanismo, que afecta a diversas fuerzas económicas y sociales relacionadas con el sector forestal. Dichas fuerzas o grupos de intereses son variados, heterogéneos y opuestos, y engloban desde el sector agrícola (a menudo importante fuerza política en los países más interesados por el mecanismo, como por ejemplo Brasil) hasta los pueblos indígenas, que aúnan cada vez más fuerzas para la defensa de sus intereses mediante la obtención de representación política a nivel regional e internacional ${ }^{13}$. En definitiva, la evolución en la configuración del mecanismo REDD+ indica que estamos ante un mecanismo cuyo alcance y efectos sobrepasan el ámbito estricto del sector forestal, por lo que se convierte en una cuestión de la agenda, más amplia, del desarrollo sostenible ${ }^{14}$.

\section{Localización del REDD+ en la estructura normativa del régimen del cambio climático}

Parte de la complejidad relacionada con el mecanismo REDD+ se deriva de la dificultad de entender su ubicación específica en el régimen del cambio climático. El mecanismo

\footnotetext{
12 A tenor no solo de los potenciales cobeneficios, sino también de los riesgos que REDD+ puede suponer para la diversidad biológica debido a la inclusión de prácticas de reforestación de especies exógenas al ecosistema forestal, el interés que despierta el mecanismo REDD+ en materia de biodiversidad se ha reflejado en su inclusión en la agenda de la COP-10 del Convenio Marco para la Protección de la Diversidad Biológica (CBD), celebrado en Nagoya en octubre del 2010. Véase la noticia relativa a la creación del REDD+ Hours en Nagoya, recuperado el 23 de octubre de 2010: http:// unredd.wordpress.com/

13 Información sobre la representación de la sociedad civil en la Cumbre de Copenhague disponible en: http://maindb.unfccc.int/public/ngo.pl (recuperado el 23 de octubre de 2010). En dicha lista aparecen 21 organizaciones catalogadas como representativas de pueblos indígenas, que fueron admitidas a participar en la Conferencia y son muestra del creciente grado de movilización de estos colectivos en los procesos de negociación internacional interestatales.
}

${ }^{14}$ Véase el Framework Document del programa UN-REDD, nota n. ${ }^{\circ} 3$ supra. 
se encuentra todavía hoy en proceso de complejización y transformación en el seno del régimen.

A partir de la adopción del Plan de Acción de Bali ${ }^{15}$ (The Bali Roadmap) en el 2007, el régimen jurídico internacional sobre el cambio climático se ha ido desarrollando por dos caminos que evolucionan paralelamente (two tracking system). Por un lado, se halla el camino que tiene por objeto la renovación de los compromisos de mitigación bajo el Protocolo de Kioto por parte de los países del anexo I para el período posterior al $2012^{16}$, año de extinción de las obligaciones recogidas en este instrumento. Dicho camino está dirigido por el grupo de trabajo ad hoc para el Protocolo de Kioto (conocido en sus siglas en inglés como AWG-KP), que la COP estableció en la sesión de Montreal del $2005^{17}$.

Por otro lado, dos años después de la sesión de Montreal, el Plan de Acción de Bali abrió un segundo camino en el seno del CMNUCC, con el objetivo de crear un protocolo bajo convenio marco que creara, entre otras cosas, compromisos de mitigación para los países en desarrollo. Este es conocido como el fututo Protocolo para la Acción Cooperativa a Largo Plazo (en adelante, Protocolo LCA). Por su parte, este segundo camino está dirigido por el grupo de trabajo ad hoc para la acción cooperativa a largo plazo (conocido en inglés como AWG-LCA) ${ }^{18}$.

Ambos caminos nacieron de modo diferenciado y se desarrollaron de manera autónoma. No obstante, en la actualidad la evolución sobre la relación de ambos caminos,

\footnotetext{
${ }^{15}$ Decisión 1/COP.13, Plan de Acción de Bali, FCCC/CP/2007/6/Add.1, p. 3, recuperado el 23 de octubre de 2010, disponible en español en:

http://unfccc.int/documentation/decisions/items/3597.php?such=i\&volltext=/CP.13\#beg

16 Protocolo al Convenio Marco de Naciones Unidas sobre el Cambio Climático, adoptado en Kioto (Japón) el 11 de diciembre de 1997.

17 Decisión-/CMP.1, "Consideration of commitments for subsequent periods for Annex I Parties to the Convention under article 3.9 of the Kyoto Protocol (KP)", Montreal (2005), recuperado el 23 de octubre de 2010, disponible en inglés en: http://unfccc.int/meetings/cop 11/items/3394.php. Información general sobre el trabajo del AWG-KP disponible en inglés en: http://unfccc.int/kyoto protocol/items/4577.php. Documentación elaborada en el seno del grupo desde el 2005 disponible en orden cronológico en: http:// unfccc.int/kyoto protocol/items/4944.php

18 Información general sobre el AWG-LCA (incluyendo la decisión 1/COP.15, por la que se aprueba el resultado del trabajo del grupo bajo el Convenio) disponible en inglés en: http://unfccc.int/meetings/ ad hoc working groups/lca/items/4381.php. Acceso a toda la documentación en orden cronológico, elaborada desde el 2007, disponible en inglés en: http://unfccc.int/meetings/ad hoc_working_groups/lca/ items/4918.php
} 
determinante de la futura arquitectura del régimen, constituye uno de los principales escollos al avance de las negociaciones. La incógnita relativa a la relación que existirá entre ambos caminos podría dar lugar a la adopción de un solo instrumento que funda ambos protocolos (el Protocolo de Kioto con el futuro Protocolo LCA), al mantenimiento de su existencia paralela y diferenciada, o bien a la adopción únicamente del Protocolo de Kioto enmendado ${ }^{19}$.

Ante esta descripción del panorama normativo del régimen de cambio climático, que permanece actualmente a la espera de cambios sustanciales en las próximas citas de las partes, es importante indicar que el mecanismo REDD+ se inscribe en el programa de trabajo sobre la acción cooperativa a largo plazo. Los textos de negociación sobre REDD+ pertenecen, por tanto, al ámbito de competencia del grupo de trabajo ad hoc correspondiente, el AWG-LCA. No obstante, previamente a la adopción de la Decisión 2/COP.13, que aceptó el mecanismo y lo inscribió en este camino, varios intentos de lograr la inclusión del sector forestal (en particular el problema de la deforestación) en la agenda del cambio climático ya habían sido emprendidos en el camino paralelo del Protocolo de Kioto ${ }^{20}$. Cabe, por tanto, abordar en la siguiente sección la distinción entre el mecanismo REDD + y las actividades de uso de la tierra, cambio de uso de la tierra y silvicultura (conocidas como LULUCF en sus siglas en inglés ${ }^{21}$ ), previamente incorporadas a los mecanismos de mercado del Protocolo de Kioto.

\section{Distinción entre el REDD+ y las actividades LULUCF incorporadas} previamente a los mecanismos flexibles del Protocolo de Kioto

\footnotetext{
${ }^{19}$ Véase TORRES CAMPRUBI, A., "Reflexiones sobre la Conferencia de Copenhague", Perspectives n. ${ }^{\circ}$ 25 (11/09), Sociedad Latinoamericana de Derecho Internacional (SLADI/LASIL), recuperado el 23 de octubre de 2010, disponible en: http://www.lasil-sladi.org/en/articulos.html. En este breve comentario sintetizo la relación de fuerzas entre distintos grupos de negociación que dificultan el acuerdo sobre la futura arquitectura del régimen tras la Cumbre de Copenhague del 2009.
}

20 Véase el informe elaborado por HOLLOWAY V. y GIANDOMENICO E., 2009, Carbon Planet White Paper: The History of REDD Policy, recuperado el 25 de octubre de 2010, disponible en: http:// www.carbonplanet.com/white papers. Este informe ofrece una línea cronológica sobre la historia del REDD+, y establece con claridad el germen preexistente en el seno del Protocolo de Kioto.

${ }^{21}$ Las siglas LULUCF se corresponden con los términos Land use, Land Use-Change and Forestry. 
Es sabido que una de las grandes innovaciones al régimen surgidas del Protocolo de Kioto fue la creación de los llamados mecanismos flexibles: el mecanismo de desarrollo limpio (MDL) y el mecanismo de aplicación conjunta (MAC). A través del MDL, los países industrializados del anexo I del Protocolo de Kioto pueden obtener créditos de cumplimiento por invertir en proyectos de reducción de emisiones localizados en países en desarrollo ${ }^{22}$. Por su parte, la AC permite que los proyectos de reducción de emisiones localizados en países del anexo I generen créditos transferibles a otros países del anexo $\mathrm{I}^{23}$.

En ambos mecanismos flexibles, el sector forestal obtuvo un espacio con anterioridad a la aprobación de la creación del mecanismo REDD+ (entonces solo conocido como RED), si bien bajo una concepción distinta de la función de este sector en el ciclo del carbono, como es la función de sumidero (carbon sinks). Esta función ya había sido recogida por el CMNUCC, que la admitía como una posible modalidad de reducción de emisiones en sus artículos 4.1.a) y 4.1.b).

De acuerdo con la definición ofrecida por el Ministerio de Medio Ambiente y Medio Rural y Marino de España, se entiende por sumidero todo sistema o proceso por el que se extrae de la atmósfera uno o varios gases que a su vez quedan almacenados ${ }^{24}$. Por tanto, a diferencia de la deforestación y la degradación forestal (fuentes de emisión de carbono), los sumideros son áreas forestales que reducen el nivel de carbono de la atmósfera, por lo que se deduce que el sector forestal tiene una relevancia de "doble cara" para el fenómeno del cambio climático. Cabe señalar que entre las perspectivas de los bosques como reservas o los bosques como fuente de emisiones de carbono, esta

\footnotetext{
${ }^{22}$ Protocolo de Kioto, nota n. ${ }^{\circ} 16$ supra, artículo 12. Para información general sobre el MDL y detalles sobre sus condiciones de operatividad, véase:

http://unfccc.int/kyoto_protocol/mechanisms/clean_development_mechanism/items/2718.php (recuperado el 23 de octubre de 2010); así como la página web específica que recoge estadísticas y novedades relativas al mecanismo flexible: http://cdm.unfccc.int/index.html (recuperado el 25 de octubre de 2010)

23 Ibíd., artículo 6. Para información general sobre el mecanismo IC, véase: http://unfccc.int/ kyoto_protocol/mechanisms/joint implementation/items/1674.php; así como la página web oficial del mecanismo donde se recoge información sobre sus novedades: http://ji.unfccc.int/index.html

${ }^{24}$ Véase nota n. ${ }^{\circ} 9$ supra.
} 
última se presenta, según lo indicado por el Cuarto Informe de Evaluación del IPCC, como la más útil al proceso global de lucha contra el cambio climático ${ }^{25}$.

La primera de las traducciones jurídicas de la función de los bosques en el ciclo del carbono, entendidos entonces como sumideros, fue la creación de la categoría de actividades de mitigación de uso de la tierra, cambio de uso de la tierra y silvicultura (en adelante, LULUCF). Muchos artículos del Protocolo de Kioto prevén la inclusión de actividades LULUCF en los esfuerzos de las partes del anexo I de cumplir con sus obligaciones de mitigación suscritas en el Protocolo ${ }^{26}$, no siendo, por tanto, de extrañar que dicha inclusión se extendiera a los mecanismos flexibles del Protocolo. La posibilidad de incorporar las emisiones LULUCF en el sistema de contabilidad de créditos de carbono se remonta, por tanto, a la Conferencia de las Partes celebrada en el 2001 en Marrakech ${ }^{27}$, cuyo propósito consistió en el desarrollo de reglas específicas para la realización de los compromisos de reducción suscritos bajo el Protocolo.

Así, por un lado, el mecanismo de desarrollo limpio permitió que las partes del anexo I del Protocolo de Kioto pudieran cumplir con sus compromisos de mitigación a través de actividades LULUCF, siendo estas igualmente útiles al desarrollo sostenible del país donde se implementa el proyecto ${ }^{28}$. No obstante, las actividades LULUCF admitidas en el marco del MDL quedaron limitadas en su alcance a actividades de forestación y reforestación ${ }^{29}$. Esta restricción dejó al margen del MDL tanto las actividades LULUCF que tuvieran por objeto el secuestro de carbono proveniente de colectas agrícolas, como los proyectos dirigidos a reducir las emisiones provenientes de la deforestación y la

\footnotetext{
25 Véase nota n. 2 supra, p. 550. El informe establece que: "la reducción de la deforestación y la degradación forestal es la opción forestal de mitigación con el mayor y más inmediato impacto en el almacenamiento (carbón stock) de carbono a corto plazo, por hectárea y por año globalmente" [traducción propia].

${ }^{26}$ Véase Protocolo de Kioto, nota n. ${ }^{\circ} 16$ supra, artículos 2.1.a.ii y 2.1.a.iii.

27 Decisión 11/COP.7, "Uso de la tierra, cambio de uso de la tierra y silvicultura", FCCC/CP/2001/13/ Add.1, recuperada el 23 de octubre de 2010, disponible en español en:
}

http://unfccc.int/documentation/decisions/items/3597.php? dec=j\&such=j\&volltext=+ $\%$ FCP\&anf $=120 \&$ sorted $=\& \operatorname{dirc}=\& a n f=120 \&$ seite $=4$

28 La promoción del desarrollo sostenible del país en desarrollo en el que se implementan proyectos MDL es consustancial a la configuración del mecanismo y criterio esencial para su admisibilidad. Véase, Protocolo de Kioto, nota n. ${ }^{\circ} 16$ supra, artículo 12.2.

${ }^{29}$ Véase nota.$^{\circ} 9$ supra. 
degradación forestal. Asimismo, las estrictas condiciones de admisibilidad de proyectos MDL establecidas en el artículo 12.5 del Protocolo de Kioto, en particular, el criterio del carácter adicional del proyecto $^{30}$, redujeron drásticamente la efectividad de los proyectos LULUCF (aun cuando estos fueran proyectos de reforestación y forestación).

Por otro lado, los proyectos LULUCF también pueden llevarse a cabo dentro del marco del mecanismo $\mathrm{AC}^{31}$, con la ventaja de no estar en principio limitados en su alcance a proyectos de reforestación y forestación dado que la AC permite la inclusión de actividades de reducción de la deforestación, de gestión forestal y de agricultura sostenible. No obstante, el interés de esta flexibilidad teórica ha quedado reducido por una interpretación restrictiva que, de hecho, impone restricciones adicionales al desarrollo de proyectos LULUCF en este mecanismo ${ }^{32}$. En consecuencia, la mayor parte de los proyectos encaminados a la reducción de la deforestación y de la degradación forestal quedan, en la práctica, igualmente excluidos del alcance de las actividades LULUCF bajo el marco de la AC.

En resumen, es importante distinguir el futuro mecanismo REDD+, dirigido a frenar la deforestación causante de emisiones de carbono y que formará parte del futuro Protocolo LCA, de las transacciones existentes en el ámbito del uso de la tierra, el cambio de uso de la tierra y la silvicultura (LULUCF), que se encuentran recogidas en el seno de los dos mecanismos flexibles (MDL y MAC) del Protocolo de Kioto. Las transacciones LULUCF en ambos mecanismos flexibles tienen por objetivo explotar y, en su caso, aumentar la capacidad de absorción de carbono de los bosques, que son considerados como reservas de carbono. En cambio, como hemos indicado, el mecanismo REDD+ se dirige, principalmente, a reducir la función de la deforestación como fuente de emisiones de carbono. Por tanto, si bien tanto el mecanismo REDD+

${ }^{30}$ El criterio de la "adicionalidad", establecido en el artículo 12.5.c) del Protocolo de Kioto, implica que el inversor debe probar que el proyecto no se presentaba como la solución más rentable, sino que su inversión fomenta la superación de algún tipo de obstáculo, por ejemplo de tipo tecnológico. Véase FAO Forest Division, Fact-sheet: Afforestation and Reforestation Projects under the Clean Development Mechanism of the Kyoto Protocol, recuperado el 23 de octubre de 2010, disponible en: www.fao.org/ forestry/8953-1-0.pdf

${ }^{31}$ Véase el Protocolo de Kioto, nota n. ${ }^{\circ} 16$ supra, artículo 6.1.b). Todos los proyectos LULUCF bajo la AC deben, no obstante, conformarse a las definiciones, reglas de contabilidad, modalidades y orientaciones establecidas en los artículos 3.3 y 3.4 del Protocolo de Kioto.

32 Véase la explicación y crítica expresadas por SCHALAMADINGER B., STRECK C. y O'SULLIVAN R., "Will Joint Implementation LULUCF projects be impossible in practice?", recuperado el 23 de octubre de 2010, disponible en www.climatefocus.org 
como los proyectos LULUCF afectan a los bosques, extraen de estos funciones distintas y se integran en dos instrumentos jurídicos diferenciados.

Ciertamente, la última extensión del alcance del mecanismo REDD+ hasta incluir el "fomento de reservas forestales de carbono" siembra confusión y ensombrece esta clara diferenciación inicial. No obstante, a pesar de este posible solapamiento, el REDD+ sigue llenando un espacio que no había sido cubierto antes por las actividades LULUCF, además de situarse en el camino de la acción cooperativa a largo plazo y no en el del Protocolo de Kioto. Llegados al punto en el que se ha expuesto qué es y qué no es el mecanismo REDD+, conviene exponer su evolución a través de las diferentes etapas de negociación.

\section{LA HISTORIA DE REDD+: PRINCIPALES ETAPAS DE SU NEGOCIACIÓN INTERNACIONAL}

\section{Bali 2007: orígenes de la propuesta de creación del mecanismo}

La propuesta de Costa Rica y Papúa Nueva Guinea sobre la creación de un mecanismo contra la deforestación fue recibido por las Partes del CMNUCC como una señal positiva de la buena disposición de los países en desarrollo de asumir esfuerzos de reducción de sus emisiones de carbono, siendo aprobada, como ya hemos indicado, por medio de la Decisión 2/COP.13 en la sesión de Bali del 2007.

Sin embargo, todo el proceso se había iniciado dos años atrás, dado que la propuesta había sido sometida a decisión de la COP en la sesión de Montreal (COP-11) del 2005. Desde aquel momento comenzó a desarrollarse el proceso — que duraría dos años- en el seno del Órgano Subsidiario de Asesoramiento Científico y Tecnológico (OSACT), que se encargó de examinar los aspectos técnicos de lo que ahora conocemos como el mecanismo REDD+, muchos de los cuales permanecen, todavía, sin elucidar.

En este período preBali, los esfuerzos de las partes se centraron en el intercambio de documentación y de información científico-técnica y consideraciones metodológicas. Se abrió igualmente el debate de las partes del Convenio, en base al cual se desarrollaron 
múltiples propuestas sobre la configuración normativa $\mathrm{y}$ financiera del futuro $\mathrm{e}$ innovador mecanismo ${ }^{33}$.

\section{Poznan 2008: el impulso del desarrollo de proyectos piloto}

En la COP-14, realizada en Poznan (Polonia) en diciembre del 2008, se impulsó la concretización de las negociaciones sobre el REDD (entonces todavía no ampliado a REDD+), con vistas a su posible adopción en la siguiente COP de Copenhague (COP-15). En Polonia, las partes revisaron las conclusiones del OSACT sobre el programa Reducción de las Emisiones derivadas de la Deforestación en los Países en Desarrollo: Métodos para Estimular la Adopción de Medidas, y recomendaron en el anexo II de estas la utilización de las directrices del IPCC sobre establecimiento de inventarios nacionales de gases de efecto invernadero, así como la promoción de sistemas nacionales transparentes e independientes de vigilancia forestal.

Bajo el impulso de los logros de Poznan, a lo largo de la primera mitad del 2009 se llevaría a cabo un gran avance del mecanismo REDD mediante la puesta en marcha de los nueve primeros proyectos piloto. Dichos proyectos fueron realizados en el marco del Programa de Colaboración de Naciones Unidas para la Reducción de las Emisiones Provenientes de la Deforestación y la Degradación Forestal en Países en Desarrollo ${ }^{34}$ (conocido en inglés como UN-REDD program), que constituye, junto con la Forest Carbon Partnership Facility del Banco Mundial ${ }^{35}$, una de las dos grandes iniciativas multinacionales destinadas a iniciar la financiación de los esfuerzos de los países interesados en "prepararse" para la futura implementación nacional de proyectos REDD.

\footnotetext{
33 Para conocer las distintas propuestas sobre la forma que debiera adoptar el mecanismo REDD realizadas por los Estados parte, por organizaciones intergubernamentales y no gubernamentales y por asociaciones corporativas, véase, Global Canopy Foundation (ed.), 2008, The Little REDD Book, recuperado el 25 de octubre de 2010, disponible en inglés en:

http://www.amazonconservation.org/pdf/redd the little redd book dec 08.pdf

34 Página web del Programa UN-REDD, recuperado el 25 de octubre de 2010, disponible en: http:// www.un-redd.org/

35 Véase página web de la Forest Carbon Partnership Facility, recuperado el 25 de octubre de 2010, disponible en: http://www.forestcarbonpartnership.org/fcp/
} 
La Junta Directiva del programa UN-REDD aprobó, así, en marzo del 2009 la asignación de fondos a Tanzania, Vietnam, Indonesia, República Democrática del Congo y Papúa Nueva Guinea para que desarrollaran sus programas nacionales $\operatorname{conjuntos}^{36}$ destinados a prepararse para $\operatorname{REDD}^{37}$. A dicha reunión, que tuvo lugar en Panamá, acudieron 50 participantes, incluyendo representantes de los países donde se están desarrollando actualmente proyectos piloto, del Gobierno de Noruega (uno de los principales Estados inversores en proyectos REDD), de organizaciones no gubernamentales, de pueblos indígenas y de las secretarías de las tres agencias de Naciones Unidas que participan actualmente en el desarrollo del mecanismo: la FAO, el Programa de Naciones Unidas para el Desarrollo (PNUD) y el Programa de Naciones Unidas para el Medio Ambiente (PNUMA) ${ }^{38}$.

\section{Copenhague 2009: avances en la operatividad del mecanismo}

La COP celebrada en Copenhague en diciembre del 2009 (COP-15) en principio debía marcar la culminación de los objetivos fijados en el 2007 por las partes en el Plan de Acción de Bali, siendo esta expectativa previa al COP-15 extensible al mecanismo REDD+. No obstante, como muchos temían a la vista del desarrollo de las consultas informales previas a la COP 15 en Bonn, Bangkok y Barcelona ${ }^{39}$, esta se cerró sin

\footnotetext{
36 Denominados en inglés National Joint Programs (NJPs). Para más información sobre los programas nacionales conjuntos, véase la siguiente página web de la UN-REDD, recuperada el 23 de octubre de 2010, disponible en:
}

http://www.un-redd.org/AboutUNREDDProgramme/NationalProgrammes/tabid/584/Default.aspx

37 Informe de la primera sesión de la Junta Directiva del Programa UN-REDD, recuperado el 23 de octubre de 2010, disponible en:

http://www.un-redd.org/PolicyBoard/1stPolicyBoard/tabid/589/language/en-US/Default.aspx

38 Esta reunión permitió realizar avances del programa UN-REDD en tres sentidos. Desde un punto de vista institucional, la Junta Directiva reafirmó la importancia de asegurar la complementariedad del Programa con otras iniciativas financieras dirigidas a la protección de los bosques, como la realizada por el Banco Mundial a través de la Forest Carbon Partnership Facility. Asimismo, se realizaron desarrollos destacables relativos al nivel de representación y participación de la sociedad civil en el programa. Así, se adoptaron los "Términos de referencia para el grupo de consulta sobre sociedad civil independiente", y se planteó la creación de un plan que permita asegurar tanto la participación de las comunidades locales y los pueblos indígenas de países en desarrollo en la toma de decisiones de actividades REDD, como el acceso directo de estos a parte de los fondos devengados.

39 Para un resumen de los avances realizados en estas sesiones, véase TORRES CAMPRUBI A. y FERNÁNDEZ EGEA R., Crónica de Derecho Internacional del Medioambiente (julio 2009-diciembre 2009), Revista Electrónica de Estudios Internacionales, n. ${ }^{\circ}$ 19/2010, recuperado el 25 de octubre de 2010, disponible en: http://www.reei.org/reei19/Cronicas_y_notas.htm 
alcanzar un acuerdo global jurídicamente vinculante, y se limitó a adoptar 23 decisiones sobre las diversas cuestiones abordadas por los dos grupos de trabajo ad hoc.

Entre las decisiones adoptadas por la COP-15 se incluye así la Decisión 4/COP-15, titulada "Orientación metodológica para las actividades destinadas a reducir las emisiones debidas a la deforestación y la degradación forestal y la función de la conservación, la gestión de bosques sostenibles y el aumento de las reservas forestales de carbono en los países en desarrollo"40. Este texto constituye el último borrador sobre el que el AWG-LCA ha estado trabajando a lo largo del 2010, con la esperanza de que el texto definitivo sea aprobado en la próxima COP-16, que tendrá lugar en Cancún el próximo diciembre del 2010.

En él se exhorta, entre otras cosas, a que los países en desarrollo identifiquen a nivel interno: 1) los agentes causantes de la deforestación en su país, así como los modos en que estos pueden ser abordados; y 2) las actividades en curso que actúen como estabilizadores de los stocks de carbono, utilizando en todo caso las orientaciones del IPCC sobre modos de estimación de los niveles de emisiones ${ }^{41}$. Asimismo, llama a los países concernidos a establecer sistemas nacionales de monitorización transparentes e incluso, en la medida en que fueran necesarios, sistemas subnacionales integrados en los sistemas nacionales ${ }^{42}$.

Por otro lado, a pesar de no haber dado lugar a un acuerdo global y jurídicamente vinculante en la Cumbre de Copenhague, la cristalización de la voluntad política quedó enmarcada en el llamado Acuerdo de Copenhague ${ }^{43}$. En él, las partes que se adhirieron al Acuerdo reiteraron la importancia crucial de crear mecanismos que incentiven la reducción de las emisiones provenientes del sector forestal de modo que se opere una

\footnotetext{
40 Decisión 4/COP.15, "Orientación metodológica para las actividades destinadas a reducir las emisiones debidas a la deforestación y la degradación forestal y la función de la conservación, la gestión de bosques sostenibles y el aumento de las reservas forestales de carbono en los países en desarrollo", FCCC/CP/ 2009/11/Add.1, p. 12, recuperado el 23 de octubre de 2010, Decisión disponible en español:

http://unfccc.int/documentation/decisions/items/3597.php?such=j\&volltext=/CP.15\#beg

${ }^{41}$ Ibíd., párrafo 1.

${ }^{42}$ Ibíd., artículo 1(h).

43 Decisión 2/COP.15, Acuerdo de Copenhague, FCCC/CP/2009/11/Add.1, p. 4, recuperado el 25 de octubre de 2010, disponible en español en:

http://unfccc.int/documentation/decisions/items/3597.php\#beg
} 
movilización de recursos de los países desarrollados a los países en desarrollo dirigidos a este fin ${ }^{44}$.

Asimismo, se menciona el sector forestal en el crucial párrafo 8 del Acuerdo de Copenhague (relativo a los compromisos sobre la futura financiación del régimen), que queda subsumido como parte de las medidas de mitigación que se beneficiarán de la movilización de 100 millones de dólares anuales hasta el 202045. Por último, si bien el Acuerdo no clarifica la cuestión del origen y la naturaleza de los fondos de mitigación dado que admite la multiplicidad de fuentes financieras ${ }^{46}$, sí establece una novedad respecto de la Conferencia de Poznan a este respecto: la creación del Fondo Verde de Copenhague para el Cambio Climático. Con independencia del origen de los recursos devengados, el Fondo Verde servirá en principio de entidad de gestión de estos para proyectos de mitigación, entre los que se incluyen los proyectos REDD $+{ }^{47}$.

Cabe recordar que el Acuerdo de Copenhague no llegó a ser adoptado como decisión de la COP, si bien la mediación del servicio jurídico del secretario general de Naciones Unidas, Ban Ki-moon, permitió salvar en cierta medida el texto, al asegurar su publicidad y permitir que los Estados que apoyaban los contenidos se inscribieran en un registro habilitado e indicaran sus medidas nacionales apropiadas de mitigación

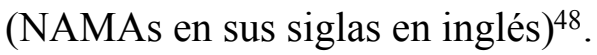

En vista de que no se logró el objetivo establecido en el Plan de Acción de Bali del 2007, la COP 15 se vio en la necesidad de prolongar el mandato de los dos grupos de trabajo ad hoc hasta la celebración de la próxima Cumbre del Clima de Cancún (México, del 29 de noviembre al 10 de diciembre de 2010). Hasta entonces, quedan por resolver cuestiones tan fundamentales como son la arquitectura legal del régimen, la escala de reducción de las emisiones (conocida como the numbers) y la financiación.

\footnotetext{
${ }^{44}$ Ibíd., párrafo 6.

45 Ibíd., párrafo 8.

46 Ibíd. El Acuerdo admite que estos puedan ser de origen público o privado, bilateral o multilateral, o bien de fuentes alternativas.

${ }^{47}$ Ibíd., párrafo 10.

48 No obstante, la no adopción del Acuerdo de Copenhague como decisión del COP afectó a la operatividad de este, pues lo relegó a un acuerdo con efectos principalmente de carácter político, sin perjuicio de que los Estados que apoyen el Acuerdo se vinculen unilateralmente a él.
} 
Frente a esta perspectiva de luces y sombras sobre el REDD+, cabe entonces preguntarse: ¿cómo funcionará el futuro mecanismo?

\section{CONCLUSIONES: INCÓGNITAS PENDIENTES SOBRE EL FUNCIONAMIENTO DEL REDD+ CON VISTAS A LA CUMBRE DEL CLIMA DE CANCÚN 2010 (COP-16)}

En la última cita de las partes celebrada en Tianjin (China) ${ }^{49}$ antes de la próxima COP-16 (Cancún, del 29 de noviembre al 10 de diciembre de 2010), la evolución de la negociación del mecanismo REDD+ parecen haberse centrado en torno a tres cuestiones, determinantes para su futura operatividad y principales obstáculos a la obtención de un acuerdo definitivo sobre el mecanismo.

Un primer escollo atañe a las consecuencias de la reciente extensión del alcance del mecanismo. Al acoger, además de la deforestación y la degradación forestal, prácticas de conservación, gestión forestal sostenible e impulso de las reservas forestales de carbono $^{50}$, queda sin esclarecer si las actividades de reducción de emisiones en terrenos no forestales como los dedicados a la agricultura quedarían igualmente cubiertas por el REDD+. No obstante, este espacio de sombra por el momento subyacente permite pensar que la eventual inclusión de estas prácticas supondría el primer paso hacia el establecimiento de un tratamiento holístico del sector forestal en el régimen normativo del cambio climático ${ }^{51}$.

\footnotetext{
${ }^{49}$ Informe completo disponible en inglés en: http://www.iisd.ca/vol12/enb12485e.html

${ }^{50}$ Véase el texto de negociación del REDD+ aprobado en Copenhague, Proyecto de Decisión -/COP.15, "Enfoques de política e incentivos positivos para las cuestiones relativas a la reducción de las emisiones debidas a la deforestación y la degradación forestal en los países en desarrollo; y la función de la conservación, la gestión sostenible de los bosques y el aumento de las reservas forestales de carbono en los países en desarrollo". Este proyecto de decisión sobre la aprobación del mecanismo pertenece al "Informe del Grupo de Trabajo Especial sobre la cooperación a largo plazo en el marco de la Convención acerca de su octavo período de sesiones, celebrado en Copenhague del 7 al 15 de diciembre de 2009", FCCC/AWGLCA/2009/17, p. 38, recuperado el 25 de octubre de 2010, disponible en:

http://unfccc.int/resource/docs/2009/awglca8/spa/17s.pdf

${ }^{51}$ En la Conferencia de Naciones Unidas sobre Medio Ambiente y Desarrollo celebrada en 1992 en Río de Janeiro, el proyecto de adoptar un convenio marco sobre bosques fracasó. Desde entonces, el sector forestal ha estado regulado a nivel internacional por la "Declaración autorizada, sin fuerza jurídica obligatoria, de principios para un consenso mundial respecto de la ordenación, la conservación y el desarrollo sostenible de los bosques de todo tipo", adoptada en la Conferencia de Río de Janeiro de 1992 y generalmente considerada un instrumento de soft law, así como por varios regímenes internacionales de medio ambiente, en particular el régimen de protección de la diversidad biológica y, más recientemente, el régimen sobre cambio climático. Véase sobre esta cuestión: HUMPHREYS D., "The elusive quest for a global forests convention", en Review of European Community and International Environmental Law, n. ${ }^{\circ} 14(1), 2005$.
} 
En este sentido, entre las propuestas presentadas se encuentran, por un lado, aquellas que abogan por un modelo de REDD+ "simple" que no trace puentes normativos con otros instrumentos en los que el sector forestal está regulado ${ }^{52}$; y por otro, aquellas que defienden que los beneficios ecológicos añadidos sean integrados en el diseño de los objetivos del REDD+ e incluidos en el cálculo de las fugas (leakages).

Un segundo punto, sin duda más controvertido y central para la futura operatividad del mecanismo, atañe a la cuestión de la repartición de papeles entre los sectores público y privado en la generación de estos nuevos recursos financieros para el REDD+. Por el momento, el párrafo 8 del Acuerdo de Copenhague parece indicar que las partes se inclinarán a adoptar un modelo de financiación mixto, mediante el cual un fondo REDD + aseguraría los recursos para la preparación de proyectos (capacity building) y se dejaría a los mecanismos de mercado la financiación de su implementación.

Si bien las futuras decisiones sobre financiación tendrán, indudablemente, un efecto directo en la efectividad del futuro mecanismo, cabe apuntar que generan asimismo un desafío de carácter ético. Hoy por hoy, las propuestas de financiación de la mayor parte de las partes y los observadores no ofrecen la posibilidad de redistribución, entre todos los actores que hayan participado en los proyectos, de los beneficios económicos derivados de la implementación de estos, incluyendo en particular a la población local ${ }^{53}$. En este sentido, es conveniente recordar que en Tianjin los países en desarrollo buscaron acercar el REDD+ a los objetivos de ayuda a la adaptación. Esta posición parece traducir la voluntad de estos países de potenciar la capacidad del REDD+ como

52 En particular, como ya hemos indicado, a través de la "Declaración autorizada, sin fuerza jurídica obligatoria, de principios para un consenso mundial respecto de la ordenación, la conservación y el desarrollo sostenible de los bosques de todo tipo", adoptada en la Conferencia de Naciones Unidas sobre Medio Ambiente y Desarrollo (Río de Janeiro), el 5 de junio de 1992; así como por el Convenio para la Protección de la Diversidad Biológica, adoptado igualmente en la Conferencia de Naciones Unidas sobre Medio Ambiente y Desarrollo (Río de Janeiro), el 5 de junio de 1992. Sobre esta cuestión, véase, por ejemplo, ROSENDAL G. K., "Overlapping international regimes: The case of the Intergovernmental Forum on Forests (IFF) between climate change and biodiversity", en International Environmental Agreements: Politics, Law and Economics, n. ${ }^{\circ} 1$ (4), 2001.

${ }^{53}$ Sin duda el mayor desafío para el mecanismo en términos de equidad se refiere al nivel de participación de las comunidades locales en los procesos de consulta previos a la elaboración de un proyecto nacional REDD+. Una de las posibilidades consistiría en que el texto REDD+ hiciera referencia a los derechos procesales de acceso a la información y consulta previa de las personas afectadas por la implementación futura. En particular, destacan la importante atención a los derechos de los pueblos indígenas, recientemente reconocidos en la Declaración de la Asamblea General de Naciones Unidas sobre los Derechos de los Pueblos Indígenas, A/61/L.67, adoptada el 11 de septiembre de 2007. 
mecanismo de ayuda al desarrollo económico y sostenible de los países donde se implanten proyectos REDD $+{ }^{54}$, e invita igualmente a revisar el importante papel de la justicia y la equidad en el seno del régimen del cambio climático.

Por último, cabe apuntar al escollo generado por la necesidad de asegurar que la certificación de créditos corresponda efectivamente a la cantidad de emisiones reducidas $^{55}$, control realizado a través de la monitorización, información y verificación (MIV) de las actividades REDD+. Hasta el momento, existe consenso sobre el ámbito que la monitorización deberá alcanzar ${ }^{56}$, pero permanece el desacuerdo en cuanto a las técnicas que se emplearán para llevar a cabo estas acciones ${ }^{57}$. La importancia de este punto de inflexión reside en que la atribución de recursos queda en todo caso supeditada a los resultados de este tipo de controles, realizados ex ante y ex post. Por un lado, los países en desarrollo tienden a proponer esquemas de MIV muy estrictos y controlados directa o indirectamente por agencias internacionales, mientras que los países en desarrollo ven en ello un riesgo o intento de "injerencia" en su soberanía ${ }^{58}$.

Estos puntos ponen de manifiesto el potencial del REDD+ para convertirse en un instrumento de "equidad interestatal", por un lado, y de promoción de la coherencia normativa intrarrégimen e interrégimen, por otro ${ }^{59}$. No obstante, con independencia de

\footnotetext{
${ }^{54} \mathrm{La}$ relación del REDD+ con la adaptación ya había sido reconocida en el párrafo 8 del Acuerdo de Copenhague, véase nota n. ${ }^{\circ} 43$ supra.

55 Véase el informe realizado por VERCHOT L. V y PETKOVA E., "The State of REDD negotiations: consensus points, options for moving forward and Research Needs to Support the Process. A Background Document for the UN-REDD Sponsored Support to Regional Groups, Center for International Forestry Research", (octubre 2009), recuperado el 25 de octubre de 2010, disponible en: http:// www.cifor.cgiar.org/Knowledge/Publications/Detail?pid=2870
}

\footnotetext{
56 Concretamente se incluiría la monitorización de: reducciones de emisiones alcanzadas en relación con las trayectorias nacionales de emisión, el costo incremental de las acciones y el desarrollo sostenible de los beneficios y cobeneficios de la implementación del REDD+.

57 Para ayudar a resolver este obstáculo, el informe de la OSCTA (nota n. ${ }^{\circ} 40$ supra) recomendó que la metodología de MIV se basara en técnicas de sensor remoto y de verificación en el terreno, y propuso el establecimiento de sistemas robustos de monitorización e inventarios nacionales forestales que permitieran revisiones periódicas de verificación de los datos. Tampoco existe hoy por hoy acuerdo sobre qué constituye un nivel de referencia, si bien ha quedado acordado que este debe estar basado en el nivel histórico de emisiones.

${ }^{58}$ Preocupación que ha sido recogida en el párrafo 2.b) del texto "Orientación Metodológica"; véase nota n. ${ }^{\circ} 40$ supra.

59 Sobre si el mecanismo alcanzará el objetivo de coherencia interrégimen, véase: LEVIN K., MCDERMOTT C. y CASHORE B., "The climate regime as global forest governance: can reduced emissions from Deforestation and Forest Degradation (REDD) initiatives pass a 'dual effectiveness' test?", en International Forestry Review, n. ${ }^{\circ} 10$ (3), 2008.
} 
la traducción jurídica final que estas dos ideas tengan en el texto definitivo sobre REDD + , no hay que olvidar que la operatividad de este depende en gran medida de que las partes alcancen un acuerdo sobre la "superestructura" en la que se inscribe, es decir, sobre el marco de la acción cooperativa a largo plazo y la ubicación de este en la arquitectura del régimen para el período posterior al 2012.

\section{DOCUMENTACIÓN}

\section{Decisiones de la Conferencia de las Partes del Convenio Marco de Naciones Unidas sobre el Cambio Climático}

Decisión 11/COP.7, "Uso de la tierra, cambio de uso de la tierra y silvicultura", FCCC/ CP/2001/13/Add.1, recuperada el 23 de octubre de 2010, disponible en español en:

http://unfccc.int/documentation/decisions/items/3597.php?dec $=\mathrm{j} \&$ such $=\mathrm{j} \&$ volltext $=+$ $\% 2$ FCP \&anf $=120 \&$ sorted $=\& \operatorname{dirc}=\& a n f=120 \&$ seite $=4$

Decisión -/CMP.1, "Consideration of commitments for subsequent periods for Annex I Parties to the Convention under article 3.9 of the Kyoto Protocol (KP)", Montreal (2005), recuperado el 23 de octubre de 2010, disponible en inglés en: http://unfecc.int/ meetings/cop_11/items/3394.php

Decisión 1/COP.13, "Plan de Acción de Bali", FCCC/CP/2007/6/Add.1, recuperado el 23 de octubre de 2010, disponible en español en:

http://unfccc.int/documentation/decisions/items/3597.php?such=j\&volltext=/CP.13\#beg

Decisión 2/COP.13, "Reducción de las emisiones derivadas de la deforestación en los países en desarrollo: métodos para estimular la adopción de medidas", FCCC/CP/ 2007/6/Add.1, recuperado el 25 de octubre de 2010, texto en español disponible en: http://unfccc.int/documentation/decisions/items/3597.php?such=j\&volltext=/CP.13\#beg Decisión 2/COP.15, “Acuerdo de Copenhague”, FCCC/CP/2009/11/Add.1, recuperado el 25 de octubre de 2010, disponible en español en:

http://unfccc.int/documentation/decisions/items/3597.php\#beg 
Decisión 4/COP.15, "Orientación metodológica para las actividades destinadas a reducir las emisiones debidas a la deforestación y la degradación forestal y la función de la conservación, la gestión de bosques sostenibles y el aumento de las reservas forestales de carbono en los países en desarrollo", FCCC/CP/2009/11/Add.1, recuperado el 23 de octubre de 2010, Decisión disponible en español:

http://unfccc.int/documentation/decisions/items/3597.php?such=j\&volltext=/CP.15\#beg

\section{Instrumentos internacionales}

Convenio Marco de Naciones Unidas sobre el Cambio Climático, adoptado en la Conferencia de Naciones Unidas sobre Medio Ambiente y Desarrollo (Río de Janeiro) entre el 4 y el 14 de junio de 1992.

Convenio para la Protección de la Diversidad Biológica, adoptado en la Conferencia de Naciones Unidas sobre Medio Ambiente y Desarrollo (Río de Janeiro) el 5 de junio de 1992.

Declaración sobre Manejo Sostenible de Bosques, adoptada en la Conferencia de Naciones Unidas sobre Medio Ambiente y Desarrollo (Río de Janeiro) el 5 de junio de 1992.

Protocolo al Convenio Marco de Naciones Unidas sobre el Cambio Climático, adoptado en Kioto (Japón) el 11 de diciembre de 1997.

Declaración de la Asamblea General de Naciones Unidas sobre los Derechos de los Pueblos Indígenas, A/61/L.67, adoptada el 11 de septiembre de 2007.

\section{Informes}

CORTEZ R. y STEPHEN P. eds. (Nature Conservacy), 2009, Introductory Course on Reducing Emissions from Deforestation and Forest Degradation and the Role of Conservation, Sustainable Management of Forest and Enhancement of Forest Carbon Stocks (REDD+): A Participant Resource Manual, (Updated April 2009), recuperado el 23 de octubre de 2010, disponible en: www.nature.org/initiatives/climatechange/files/ redd training_manual.pdf 
FAO Forest Division, Fact-sheet: Afforestation and Reforestation Projects under the Clean Development Mechanism of the Kyoto Protocol, recuperado el 23 de octubre de 2010, disponible en: www.fao.org/forestry/8953-1-0.pdf

GLOBAL CANOPY FOUNDATION (ed.), 2008, The Little REDD Book, recuperado el 25 de octubre de 2010, disponible en inglés en:

http://www.amazonconservation.org/pdf/redd the little_redd_book_dec 08.pdf HOLlOWAY V. y GIANDOMENICO E., 2009, Carbon Planet White Paper: The History of REDD Policy, recuperado el 23 de octubre de 2010, disponible en: http:// www.carbonplanet.com/white_papers

IPCC, 2007, Cuarto Informe de Evaluación (Fourth Assessment Report), recuperado el 25 de octubre de 2010, disponible en inglés en: http://www.ipcc.ch/pdf/assessmentreport/ar4/syr/ar4 syr.pdf

IPCC, 2007, Forestry in Climate Change 2007: Mitigation. Contribution of Working Group III to the Fourth Assessment Report of the Intergovernmetnal Panel on Climate Change, recuperado el 25 de octubre de 2010, disponible en: http:/www.ipcc.ch/ ipccreports/ar4-wg3.htm

SCHALAMADINGER B., STRECK C. y O'SULLIVAN R., "Will Joint Implementation LULUCF projects be impossible in practice?", recuperado el 23 de octubre de 2010, disponible en: $\underline{w w w . c l i m a t e f o c u s . o r g}$

UN-REDD Program, 2008, Framework Document, recuperado el 25 de octubre de 2010, disponible en: http://www.un-redd.org/Publications/tabid/587/Default.aspx 